\title{
Scalable Approach for Continuous-Wave Deep-Ultraviolet Laser at 213nm
}

\author{
Yushi Kaneda $^{1,2}$, Tsuyoshi Tago ${ }^{1}$, Toshiaki Sasa ${ }^{1}$, Masahiro Sasaura ${ }^{1}$, Hiroaki Nakao ${ }^{1}$, Junji Hirohashi ${ }^{1}$, and \\ Yasunori Furukawa ${ }^{1}$ \\ ${ }^{1}$ Oxide Corporation, 1747-1 Mukawa, Hokuto, Yamanashi 408-0302, JAPAN \\ ${ }^{2}$ University of Arizona, College of Optical Sciences, 1630 E University Blvd., Tucson, AZ 85721 USA \\ kaneda@opt-oxide.com
}

\begin{abstract}
We present a novel approach for generation at $213 \mathrm{~nm}$, corresponding to the fifth harmonic of common $1064 \mathrm{~nm}$ laser, in pure continuous-wave mode. Starting from two infrared fiber laser sources, we demonstrated $0.45 \mathrm{~W}$ output at $213 \mathrm{~nm}$. (c) 2018 The Author(s)

OCIS codes: (140.3610) Lasers, ultraviolet; (140.3515) Lasers, frequency doubled; (190.2620) Harmonic generation and mixing;
\end{abstract}

Continuous-wave (CW) fourth-harmonic laser sources at $266 \mathrm{~nm}$, the fourth harmonic wavelength, being industrially deployed in a variety of applications. Now, CW laser sources at $213 \mathrm{~nm}$, a natural next step at the fifth harmonic wavelength, had long been sought for. Demand for shorter wavelengths and higher output powers seems persistent, for example, for higher throughput or for higher $\mathrm{S} / \mathrm{N}$ ratio in the inspection of semiconductor which keeps shrinking in size. In order to generate $213 \mathrm{~nm}$, conventionally the forth harmonic of $1064 \mathrm{~nm}$ is frequency mixed with the fundamental [1]. However, this approach requires the forth harmonic, which is already in the deep UV, to be tightly focused into the nonlinear crystal, hence limiting the operational lifetime of the crystal. Further, the output power will be limited by the availability of the fourth harmonic power.

For the objective of stably generating higher powers at $213 \mathrm{~nm}$, we decided to take a different approach [2]; $213 \mathrm{~nm}$ is generated by the second harmonic generation (SHG) of $426 \mathrm{~nm}$, which can be obtained by the second harmonic of $852 \mathrm{~nm}$. To generate high-power at $852 \mathrm{~nm}$, we chose the sum-frequency mixing as the scalable approach; $1907 \mathrm{~nm}$ from a thulium-doped fiber source and 1540nm from an erbium-doped fiber source are employed to efficiently generate $852 \mathrm{~nm}$ by doubly-resonant sum-frequency mixing (DRSFM). Generated $852 \mathrm{~nm}$ is subsequently converted to $213 \mathrm{~nm}$ by cascaded external resonant doublers. The resulting output is purely continuous and in single frequency. Fig. 1 shows the schematic of our approach.

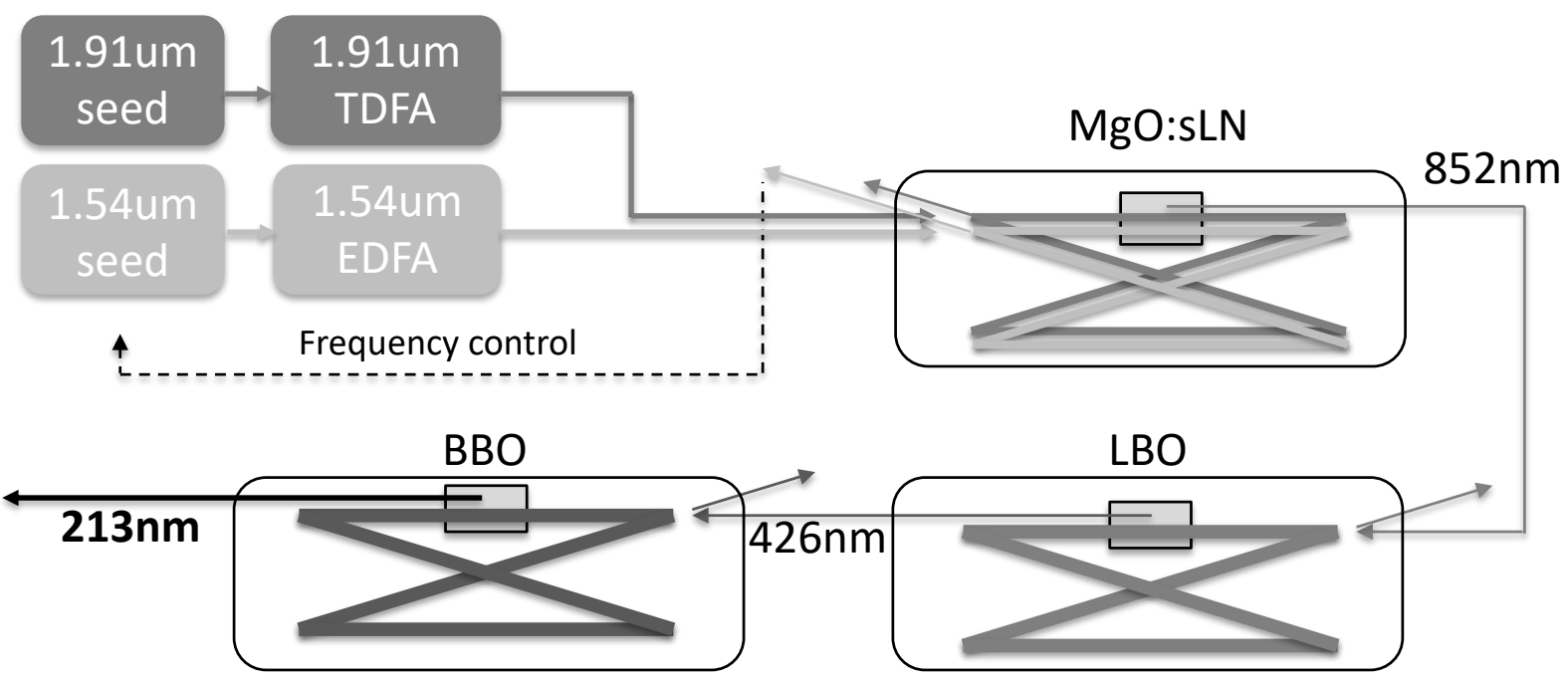

Fig. 1. Schematic of the continuous-wave $213 \mathrm{~nm}$ laser

The thulium-doped fiber amplifier (TDFA) is an in-house, single-stage clad-pumped amplifier, and is seeded by an in-house, single-frequency fiber laser oscillator at $1907 \mathrm{~nm}$ with an output power of $40 \mathrm{~mW}$. Pumped with two 9-W multimode diode lasers at $793 \mathrm{~nm}$, it gives an output power of approximately $5 \mathrm{~W}$. The seed laser at $1907 \mathrm{~nm}$ is 
free running and does not have the frequency control capability. A commercial erbium-doped fiber amplifier (IPG Photonics) has the maximum output power of $10 \mathrm{~W}$ and is seeded by a commercial single-frequency fiber laser with frequency control capability (NKT Photonics). The DRSFM cavity is locked to the free-running 1907nm, and the frequency of the $1540 \mathrm{~nm}$ is locked to the cavity, hence realizing simultaneous resonance of two wavelengths [3]. A Brewster-cut MgO-doped periodically-poled stoichiometric lithium tantalate poled at $25.37 \mu \mathrm{m}$ for the first order quasi-phasematching for SFM is placed at the waist of the cavity. With the maximum available input from two fiber sources, a maximum output power of $5.2 \mathrm{~W}$ of $852 \mathrm{~nm}$ was observed. Since all 3 interacting beams are all in the same polarization, the Brewster-cut end facets are effective for low loss resonator as well as for low-loss outcoupling of the generated $852 \mathrm{~nm}$. With up to $4.02 \mathrm{~W}$ of $852 \mathrm{~nm}$ available for the harmonic generation, $2.41 \mathrm{~W}$ of $426 \mathrm{~nm}$ was obtained from the first external resonant doubler containing $20 \mathrm{~mm}$ long type-I LBO cut at $\varphi=90^{\circ}$, $\theta=27.4^{\circ}$ for the type-I critical phasematching at room temperature.

For the harmonic generation at $213 \mathrm{~nm}, \mathrm{BBO}$ is practically the only choice as the nonlinear material. We used a Brewster-cut, $10 \mathrm{~mm}$ long, Czochralski-grown BBO crystal cut at $\theta=72.9^{\circ}$ for the type-I phasematched SHG at $213 \mathrm{~nm}$ [4]. The second resonant doubler has the waist of $44 \mu \mathrm{m}$ (non-walkoff direction) and $35 \mu \mathrm{m}$ (walkoff direction) in radius. We estimate the normalized conversion efficiency to be approximately $0.5 \times 10^{-4} \mathrm{~W}^{-1}$. The generated $213 \mathrm{~nm}$ light is outcoupled by a dichroic Brewster beamsplitter; a fused silica plate with the angle of incidence of $56^{\circ}$ with the first surface coated for high reflection at $213 \mathrm{~nm}$ in s-polarization and high transmission at $426 \mathrm{~nm}$ in p-polarization. With $2.41 \mathrm{~W}$ at $426 \mathrm{~nm}$ incident onto the second doubler, more than $0.45 \mathrm{~W}$ of $213 \mathrm{~nm}$ was observed. The input-output characteristics of $213 \mathrm{~nm}$ generation is shown in Fig. 2.

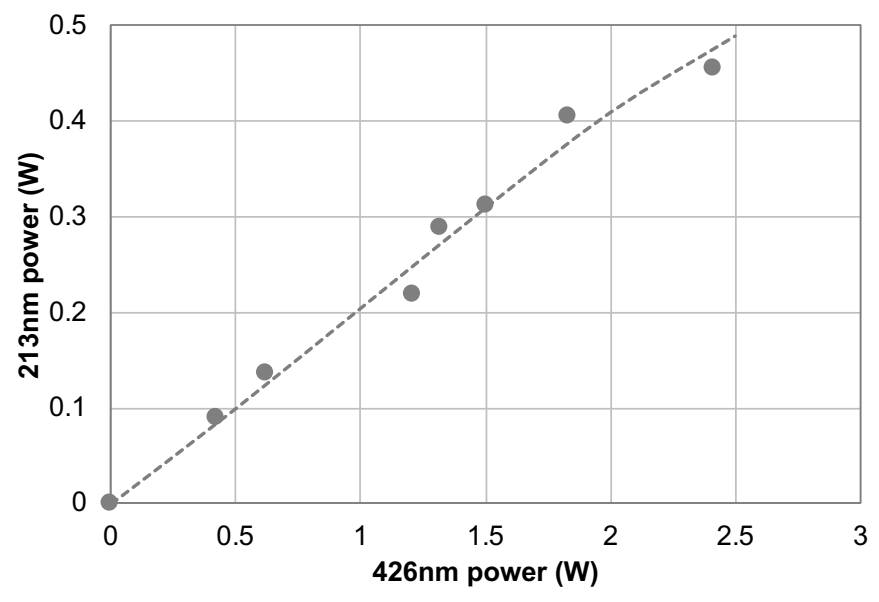

Fig. 2. Input-Output characteristics of $213 \mathrm{~nm}$ generation

We believe this is the highest output power of CW $213 \mathrm{~nm}$ reported so far to the best of our knowledge. The output powers from the fiber sources at both $1540 \mathrm{~nm}$ and $1907 \mathrm{~nm}$ are readily scalable. With higher output powers at $852 \mathrm{~nm}$, we believe the $\mathrm{CW} 213 \mathrm{~nm}$ output is also scalable.

We gratefully acknowledge the support from New Energy and Industrial Technology Development Organization (NEDO) of Japan.

\section{References}

[1] J. Sakuma, Y. Asakawa, T. Imahoko, and M. Obara, "Generation of all-solid-state, high-power continuous-wave 213-nm light based on sumfrequency mixing in $\mathrm{CsLiB}_{6} \mathrm{O}_{10}$," Opt. Lett. 29, 1096-1098 (2004).

[2] Y. Kaneda, U.S. Patent 9,429,813 “Deep ultraviolet laser generation device and light source device,” (2016).

[3] Y. Kaneda, and S. Kubota, "Theoretical treatment, simulation, and experiments of doubly resonant sum-frequency mixing in an external resonator," Appl. Opt. 36, 7766-7775 (1997).

[4] K. Kato, N. Umemura, and T. Mikami, "Sellmeier and thermo-optic dispersion formulas for $\beta$-BaB2O4 (revisited)," Proc. SPIE 7582, 75821L (2010). 